\section{Using Advanced Optical Sensing to Quantify Phytotoxicity in Ornamental Plants}

\author{
Christian Nansen ${ }^{1}$, Rachel Purington ${ }^{1}$, and Machiko Murdock ${ }^{1}$
}

ADDITIONAL INDEX WORDS. crop stress, machine vision and automation, remote sensing, visual inspection

SuMMARY. Ornamental crop production systems face low tolerance of aesthetic crop damage by consumers and during exports by quarantine inspection entities.

Consequently, development and testing of pesticide applications on ornamental crops involve two equally important assessments: 1 ) demonstrate the ability of pesticides to suppress target pest populations significantly and 2) minimize risks of applied pesticides causing phytotoxicity of leaves, shoots, and flowers. To maximize the accuracy and repeatability of phytotoxicity assessments, it is paramount that methods of detection and diagnosis that are rapid, repeatable, and quantitative be developed and promoted. We performed visual phytotoxicity inspection of three ornamental plants [zinnia (Zinnia elegans), marigold (Tagetes patula), and gerbera (Gerbera sp.)] to a numbered compound applied at three doses. The same plants were also subjected to optical (remote) sensing and classified as having either no or low phytotoxicity response. Although results from visual inspections suggested very low levels of phytotoxicity, 32 of 40 plants $(80 \%)$ were classified correctly based on optical sensing. Importantly, classified plants showed no significant morphometric differences. We provide proof-ofconcept results that optical sensing may be used to detect accurately even highly subtle stress responses by ornamental plants to high doses of foliar pesticides.

$\mathrm{I}$ general, pesticides are applied to ornamental plants to protect against crop damage by insects, diseases, and additional plant pests, but it is equally important that pesticide applications themselves do not adversely affect commercialization of ornamental crop plants. "Phytotoxicity" is defined as the temporary or longlasting effects of a compound (e.g., a pesticide) on plants (European and Mediterranean Plant Protection Organization, 2014). Above levels causing

Received for publication 8 Apr. 2021. Accepted for publication 12 May. 2021.

Published online 23 June 2021.

${ }^{1}$ Department of Entomology and Nematology, Uni versity of California, Davis, Briggs Hall, Room 367, Davis, CA 95616

This study was supported in part by grants from the U.S. Department of Agriculture (USDA), Agricultural Marketing Service through grant 18-00001056-SC; the USDA, IR4 program; the American Floral Endowment; the Gloeckner Foundation; and the USDA, Agricultural Research Service, Floriculture, Nursery Research Initiative.

University of California, Davis is an equal opportunity employer and service provider. Any opinions, findings, conclusions, or recommendations expressed in this publication are those of the authors and do not necessarily reflect views held by the University of California, Davis.

C.N. is the corresponding author. E-mail: chrnansen@ ucdavis.edu.

This is an open access article distributed under the CC BY-NC-ND license (https://creativecommons. org/licenses/by-nc-nd/4.0/).

https://doi.org/10.21273/HORTTECH04866-21 plant stress, reduction in vigor, and reduction in aesthetic value, phytotoxicity may affect the marketability of ornamental crops adversely. The main purpose of our study was to examine challenges with conventional phytotoxicity assessment practices. We present proof of concept of optical (remote) sensing as a method to improve and automate phytotoxicity assessments of ornamental crops.

Assessment of phytotoxicity in ornamental plants is typically based on visual inspection of leaves, shoots, or flowers at different time points after plants were sprayed with pesticides, and individual plants are scored according to a severity scale. A representative example of phytotoxicity assessment in a variety of fern species (Polypodiopsida) used the following scaling (Stamps et al., 2004): 1 = normal; 2 = slight damage, saleable; 3 $=$ moderate damage, unsaleable; $4=$ of phytotoxicity in roses (Rosa sp.), specific scales were used for shoots $(0=$ no visible injury, $1=1 \%$ to $5 \%$ shoot damage, $2=6 \%$ to $10 \%$ shoot damage, and 3 severe damage; and $\mathbf{5}=$ dead. In a study
$=$ more than $10 \%$ shoot damage $)$ and flowers $(0=$ no damage; $\mathrm{l}=$ minor burning of leaves, stems, and petals; and $2=$ major burning of leaves, stems, and petals) (Damte, 2019). As pointed out by Nash (1981) and many others, visual assessments of phytotoxicity are 1) subjective and therefore not easy to perform consistently and with repeatable results, 2) highly influenced by experience and training of the person performing assessments, 3 ) time-consuming if performed carefully, and 4) difficult to translate among species of ornamental plant species and/or between plants in different developmental stages. As an example of the latter point, flower phytotoxicity in gerbera (Gerbera sp.) and roses treated with the same product and dose are difficult to compare directly, because of differences in numbers of flower heads and leaves per plant. That is, conversion of assessments of phytotoxicity into a percentage ignores important differences in total number of leaves, shoots, or flowers among plants. Also, regarding leaf phytotoxicity, an accurate percentage can only be estimated if virtually all leaves on all plants are counted, which is not practically feasible for most ornamental plants. A final point of concern relates to the actual phytotoxicity symptom, which typically varies in degree of severity. It may just be a subtle curling or burning of leaves, or it may be distinct spots of chlorosis or even dead leaf tissue. Thus, in addition to distribution and abundance of signs of phytotoxicity, the assessment should ideally also encompass some measurement of severity/intensity.

\section{Data supporting proof of concept}

We present data from a study in which the purpose was to assess possible phytotoxicity by a numbered compound, V-10433, on three horticultural crops: zinnia (Zinnia elegans), marigold (Tagetes patula), and gerbera. For each ornamental crop, three doses were applied as foliar sprays (low, 0.86 $\mathrm{mL} \cdot \mathrm{L}^{-1} ;$ medium, $1.72 \mathrm{~mL} \cdot \mathrm{L}^{-1}$; and high, $3.44 \mathrm{~mL} \cdot \mathrm{L}^{-1}$ ) as well as water only (positive control) to 10 plants of each species assigned to each treatment. Leaf phytotoxicity was based on a scale from 0 to $10 \quad(0=$ no

\begin{tabular}{llll}
\hline $\begin{array}{l}\text { Units } \\
\text { To convert U.S. to SI, } \\
\text { multiply by }\end{array}$ & U.S. unit & SI unit & $\begin{array}{l}\text { To convert SI to U.S., } \\
\text { multiply by }\end{array}$ \\
\hline 0.001 & $\mathrm{ppm}$ & $\mathrm{mL} \cdot \mathrm{L}^{-1}$ & 1000
\end{tabular}


phytotoxicity, $10=$ dead plant), with principal symptoms of phytotoxicity being varying degrees of browning/ discoloring of leaf edges. Phytotoxicity of flowers was scored as presence ( 1 ) or absence $(0)$, but is not presented here. Leaf phytotoxicity was assessed at seven time points [days after treatment (DAT) ]: -1 (before spray applications), $6,13,20,27,34$, and 41 DAT. Two morphometric traits-height (longest vertical distance from base of individual plants to tip of leaf) and width (longest horizontal distance across individual plants) - were measured for all plants at all time points. Regarding zinnia, leaf toxicity was not observed for any of the 28 combinations of DAT and treatments (control and low, medium, and high dose). In addition, only one zinnia plant showed subtle signs of leaf phytotoxicity (score, 1 ) to a high dose at 20 DAT. Regarding marigolds at 20 DAT, a leaf phytotoxicity score of 1 was assigned to 4 and 5 of the 10 plants under medium- and high-dose treatments, respectively. No leaf phytotoxicity was observed on plants assigned to control or low-dose treatments. The slight difference in phytotoxic responses among ornamental plants emphasizes the importance of testing doses of specific pesticides on multiple plant species. Based on pairwise Tukey comparisons of average data from marigold plants subjected to the four treatments (control and low, medium, and high dose), we observed no significant effects on height and width of plants, and no significant effect on average number of flowers $(P>0.05)$. These morphometric plant data underscore that observed leaf phytotoxicity stress responses were highly subtle. A leaf phytotoxicity score of $l$ is very low and is unlikely to affect marketability of such ornamental plants, but this dataset was considered highly suitable for testing the proof of concept of using optical sensing to detect leaf phytotoxicity accurately. That is, one would expect a strong positive association between level of leaf phytotoxicity stress expression and the accuracy of detection based on leaf reflectance. Thus, possible detection of leaf phytotoxicity of marigold plants subjected to medium and high doses with leaf phytotoxicity scores of 1 would indicate a high degree of both sensitivity and accuracy of optical sensing technologies. Furthermore, assessment of phytotoxic responses based on classification of optical sensing data represents a way to automate plant inspections and therefore increase the consistency of results.

\section{Optical sensing of phytotoxicity}

Although visual inspection will probably remain a critical component of phytotoxicity assessments, factors such as labor shortages and operational costs are driving a growing demand for automation of agricultural practices. Automation may include crop monitoring, and detection and diagnosis of arthropod pest outbreaks (Jha et al., 2019; Shamshiri et al., 2018). Advanced optical sensing technologies can be used to detect subtle plant responses to both abiotic and biotic stressors (Behmann et al., 2015; Lowe et al., 2017; Nansen and Elliott, 2016; Prabhakar et al., 2012). Using a robotic optical sensing system inside a dark room and with controlled artificial lighting, hyperspectral imaging data were acquired from ornamental plants (Fig. 1A). Only data from marigold plants at 20 DAT are presented here, because they exhibited subtle but consistent phytotoxic stress responses to medium and high doses of the foliar pesticide. Figure IB presents a representative example of 10 marigold plants as imaged with the hyperspectral optical sensing system. Before data analyses, optical sensing data were spatially binned (spatial resolution was reduced) 900 times by $30 \times 30$ averaging of pixels (Fig. 1C). Spatial binning enhances smoothing of hyperspectral data (decreases levels of stochastic radiometric noise) and may also increase the accuracy of pixel classifications (Nansen et al., 2013). To illustrate phytotoxic stress responses, average reflectance profiles from treated plants were divided by average leaf reflectance profiles from control plants. Thus, spectral band values near $\mathrm{l}$ (dotted horizontal line in Fig. 1D) imply a high degree of similarity, and therefore no signs of phytotoxicity. Values $>1$ imply a greater leaf reflectance of treated plants compared with positive control plants, and $<\mathrm{l}$ imply lower leaf reflectance of treated plants compared with positive control plants. Figure ID presents data that a high-dose treatment of marigold plants caused a marked increase in leaf reflectance in spectral bands from 750 to $1015 \mathrm{~nm}$, whereas leaf reflectance in this spectral range of marigold plant subjected to low and medium doses was similar to those of control plants.
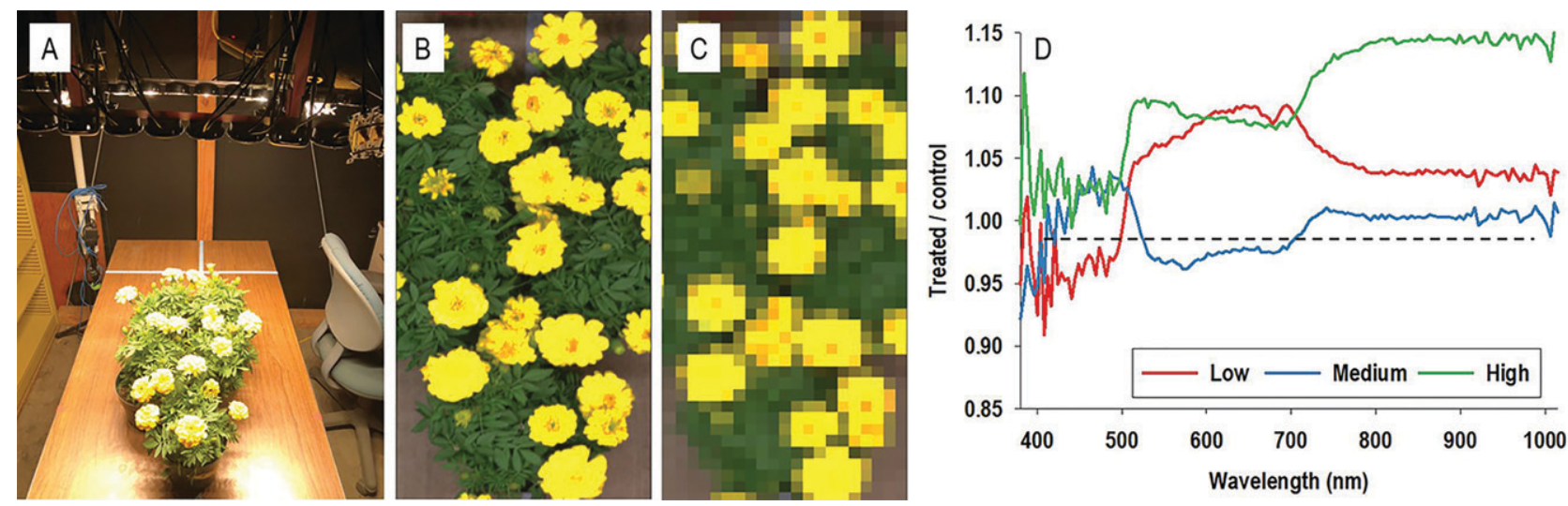

Fig. 1. (A) Ornamental crop plants subjected to hyperspectral optical sensing. (B) A representative image of marigold plants imaged. (C) The same image after $30 \times 30$ spatial binning of pixels. (D) Average leaf reflectance from marigolds after leaf reflectance data from low-, medium-, and high-dose treatments were divided with those from control plants. Curves show the relative treatment effect, with the dotted horizontal line denoting no treatment effect (no difference in average leaf reflectance from that of control plants). 
Table 1. Confusion matrix (classification results) of spatially binned pixels from marigold plants acquired $20 \mathrm{~d}$ after treatment and assigned to one of two classes based on visual inspection: no or low leaf phytotoxicity.

\begin{tabular}{lccc}
\hline & \multicolumn{3}{c}{ Predicted/classified class $^{\mathbf{y}}$} \\
\cline { 2 - 4 } Actual class $^{\mathbf{z}}$ & No & Low & Accuracy (\%) $^{\mathbf{x}}$ \\
\hline No phytotoxicity & $\mathbf{5 1 5}$ & 234 & 68.76 \\
Low phytotoxicity & 260 & $\mathbf{5 0 6}$ & 66.06 \\
\hline
\end{tabular}

${ }^{\mathrm{z}}$ In total, 1515 pixels were acquired from marigold plants at $20 \mathrm{~d}$ after treatment, and these plants were assigned leaf phytotoxicity scores of 0 (no phytotoxicity) or 1 (low phytotoxicity) based on visual inspection.

${ }^{\mathrm{y}}$ Machine learning (support vector machine) was used to classify individual pixels into the two classes. Numbers in bold denote correctly classified pixels for each of the two classes.

${ }^{\mathrm{x}}$ Accuracy denotes the percentage of pixels of each class that were classified correctly.

After spatial binning of optical sensing data and removal of background (table and pot) and pixels denoting flowers, $\approx 35$ pixels represented leaf reflectance profiles from each of the 40 marigold plants (10 from each of four treatments). Data processing and analyses were conducted in $\mathrm{R}$ (version 3.6.1; R Foundation for Statistical Computing, Vienna, Austria), and we used the "el07l" library to perform support vector machine (Cortes and Vapnik, 1995) with linear kernel classification. Before analysis, plants were grouped into "no phytotoxicity response" (control and low-dose plants) and "low phytotoxicity response" (medium- and high-dose plants). Pixels from each class were classified with $69 \%$ (no) and 66\% (low) accuracy, respectively (Table 1 ). With $\approx 35$ pixels classified from each plant and each pixel classified individually, the actual assignment of plants to one of the two classes is based on greatest number of pixels. Thus, with a classification accuracy of $67 \%, 33 \%$ of pixels may be mis-classified as the wrong class, but any given plant is likely assigned to the correct class. We confirmed this by determining how each plant was classified: 1) of control and low-dose plants, seven and eight plants were classified correctly as no phytotoxicity, respectively; and 2) of medium- and high-dose plants, eight and nine plants were classified correctly as low phytotoxicity. In total, 32 of 40 plants $(80 \%)$ were classified correctly. The potential of optical sensing technology is underscored by the fact that it was possible to distinguish marigold plants with either no or only subtle signs of leaf phytotoxicity.

\section{Conclusion}

To maximize accuracy and repeatability of phytotoxicity assessments, it is paramount to develop and promote methods of detection and diagnosis that are more rapid, repeatable, quantitative, and reliable. The proof-of-concept results presented here support the claim that optical sensing may be used to detect accurately even highly subtle stress responses by ornamental plants to high doses of foliar pesticides. It is important to mention that we classified plants in two classes with no significant morphometric differences and with only very subtle differences in phytotoxicity response. It was beyond the scope of this study to characterize to what extent the phytotoxic stress responses by ornamental plants may be different from stress responses to abiotic (e.g., irrigation, fertilizer) and biotic (e.g., root nematodes, insect, diseases) plant stressors. Few optical sensing studies involve the diagnosis of stress responses to one of several stressors, but a recent study demonstrated that leaf miner (Liriomyza trifolii) infestations in bok choy (Brassica rapa var. chinensis) and 'Correnta' spinach (Spinacia oleracea) could be detected accurately over time and across fertilizer regimes (Nguyen and Nansen, 2020). Additional research into phytotoxicity responses by ornamental plants is needed, and it is of particular importance that such studies focus on ways to acquire and classify optical sensing data so that phytotoxic stress responses can be separated accurately from those elicited by both single factors and combinations of factors, such as biotic stressors, physical damage to plants, and stress induced by suboptimal growing conditions (abiotic factors).

\section{Literature cited}

Behmann, J., A.-K. Mahlein, T. Rumpf, C. Römer, and L. Plümer. 2015. A review of advanced machine learning methods for the detection of biotic stress in precision crop protection. Precis. Agr. 16:239-260.

Cortes, C. and V. Vapnik. 1995. Supportvector networks. Mach. Learn. 20:273-297.
Damte, T.B. 2019. Phytotoxicity of tracer 120SC on greenhouse-grown rose (Rosa sp.) flowers. Arch. Phytopathol. Pflanzenschutz 52:114-124, doi: https://doi. org/10.1080/03235408.2019.1585408.

European and Mediterranean Plant Protection Organization. 2014. PP 1/135 (4) Phytotoxicity assessment. OEPP. Bull. OEPP 44: 265-273, doi: https://doi.org/10.1111/ epp.12134.

Jha, K., A. Doshi, P. Patel, and M. Shah. 2019. A comprehensive review on automation in agriculture using artificial intelligence. Artif. Intel. Agr. 2:1-12, doi: https://doi. org/10.1016/j.aiia.2019.05.004.

Lowe, A., N. Harrison, and A.P. French. 2017. Hyperspectral image analysis techniques for the detection and classification of the early onset of plant disease and stress. Plant Methods 13:80, doi: https://doi. org/10.1186/s13007-017-0233-z.

Nansen, C. and N. Elliott. 2016. Remote sensing and reflectance profiling in entomology. Annu. Rev. Entomol. 61:139-158, doi: https://doi.org/10.1146/annurevento-010715-023834.

Nansen, C., L.D. Geremias, Y. Xue, F. Huang, and J.R. Parra. 2013. Agricultural case studies of classification accuracy, spectral resolution, and model over-fitting. Appl. Spectrosc. 67:1332-1338, doi: https://doi. org/10.1366/12-06933.

Nash, R.G. 1981. Phytotoxic interaction studies: Techniques for evaluation and presentation of results. Weed Sci. 29:147-155

Nguyen, H. and C. Nansen. 2020. Hyperspectral remote sensing to detect leafminer-induced stress in bok choy and spinach according to fertilizer regime and timing. Pest Mgt. Sci. 76:2208-2216, doi: https://doi.org/10.1002/ps.5758.

Prabhakar, M., Y.G. Prasad, and M.N. Rao. 2012. Remote sensing of biotic stress in crop plants and its applications for pest management, p. 517-549. In: B. Venkateswarlu, A.K. Shanker, C. Shanker, and M. Maheswari (eds.). Crop stress and its management: Perspectives and strategies. Springer, New York, NY.

Shamshiri, R.R., F. Kalantari, K.C. Ting, K.R. Thorp, I.A. Hameed, C. Weltzien, D. Ahmad, and Z.M. Shad. 2018. Advances in greenhouse automation and controlled environment agriculture: A transition to plant factories and urban agriculture. Intl. J. Agr. Biol. Eng. 11:1-22, doi: https://doi. org/10.25165/j.ijabe.20181101.3210.

Stamps, R.H., A.L. Chandler, and D.K. Rock. 2004. Phytotoxicity evaluation of milbemectin on ferns. Proc. Florida State Hort. Soc. 117:309-312. 